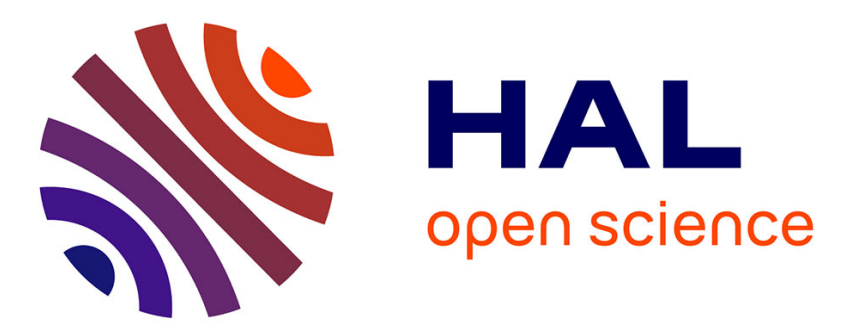

\title{
La modélisation de l'évolution du contrôle de gestion dans une population d'organisations : le cas des caisses primaires d'assurance maladie (CPAM)
}

Laurent Spang

\section{- To cite this version:}

Laurent Spang. La modélisation de l'évolution du contrôle de gestion dans une population d'organisations: le cas des caisses primaires d'assurance maladie (CPAM). La Revue des Sciences de Gestion, 2011, 5 (251), pp.145-156. 10.3917/rsg.251.0145 . hal-03028258

\section{HAL Id: hal-03028258 \\ https://hal.science/hal-03028258}

Submitted on 27 Nov 2020

HAL is a multi-disciplinary open access archive for the deposit and dissemination of scientific research documents, whether they are published or not. The documents may come from teaching and research institutions in France or abroad, or from public or private research centers.
L'archive ouverte pluridisciplinaire HAL, est destinée au dépôt et à la diffusion de documents scientifiques de niveau recherche, publiés ou non, émanant des établissements d'enseignement et de recherche français ou étrangers, des laboratoires publics ou privés. 


\section{LA MODÉLISATION DE L'ÉVOLUTION DU CONTRÔLE DE GESTION DANS UNE POPULATION D'ORGANISATIONS : LE CAS DES CAISSES PRIMAIRES D'ASSURANCE MALADIE (CPAM)}

\section{Laurent Spang}

Direction et Gestion | « La Revue des Sciences de Gestion »

2011/5 n² 251 | pages 145 à 156

ISSN 1160-7742

ISBN 9782916490304

Article disponible en ligne à l'adresse :

https://www.cairn.info/revue-des-sciences-de-gestion-2011-5-page-145.htm

Distribution électronique Cairn.info pour Direction et Gestion.

(C) Direction et Gestion. Tous droits réservés pour tous pays.

La reproduction ou représentation de cet article, notamment par photocopie, n'est autorisée que dans les limites des conditions générales d'utilisation du site ou, le cas échéant, des conditions générales de la licence souscrite par votre établissement. Toute autre reproduction ou représentation, en tout ou partie, sous quelque forme et de quelque manière que ce soit, est interdite sauf accord préalable et écrit de l'éditeur, en dehors des cas prévus par la législation en vigueur en France. Il est précisé que son stockage dans une base de données est également interdit. 


\title{
La modélisation de l'évolution du contrôle de gestion dans une population d'organisations : le cas des Caisses Primaires d'Assurance Maladie (CPAM)
}

\author{
par Laurent Spang
}

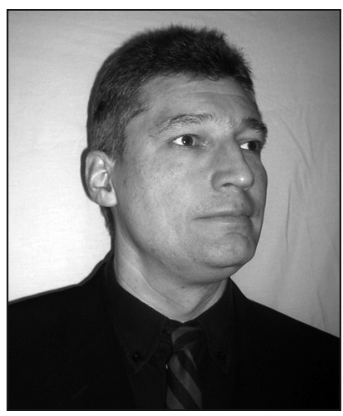

Laurent SPANG

Maître de conférences en Sciences Économiques

Docteur en Sciences de gestion

Responsable du M2 "Chargé d'études

économiques, développement local, contrôle organisationnel

UFR Droit, Économie, Administration de Metz. France 'objet de la recherche concerne le domaine de l'Assurance maladie et, plus précisément, les CPAM (Caisses Primaires d'Assurance Maladie). Les CPAM sont des organismes situés au bas de la pyramide de la Sécurité sociale où elles sont en contact direct avec le public. Notons que si elles gèrent localement, pour le régime général, la branche maladie, elles gèrent également la branche accident du travail. II y a 128 CPAM. Ce sont des organisations privées ayant une mission de service public. De ce fait, elles présentent à la fois des caractéristiques du secteur privé et du service public. Les CPAM s'occupent de l'immatriculation des assujettis, du versement des prestations, de l'action sanitaire et sociale (campagnes de prévention), de la maîtrise médicalisée des dépenses, etc. Ces activités sont obligatoires, elles sont imposées aux CPAM par l'État. La CNAMTS (Caisse Nationale d'Assurance Maladie des Travailleurs Salariés) est l'organisme qui chapeaute les CPAM et leur alloue des ressources. Elle joue le rôle de tête de réseau. Elle a mis en place un système de benchmarking entre les CPAM.

L'Assurance maladie, du fait des déficits qui s'y développent, attire depuis plusieurs années l'attention des médias. Ces déficits, bien qu'essentiellement exogènes à la gestion des caisses, sont à l'origine d'une tendance à la rationalisation de cette gestion; c'est ainsi que s'est créé un contexte favorable à l'émergence et au développement du contrôle de gestion. Nous définirons le contrôle de gestion comme "le processus par lequel les managers influencent d'autres membres de l'organisation pour appliquer les stratégies" (R.N. Anthony, 1988 in H. Bouquin, 1967 p. 18). Dans la population des CPAM, le principe de management étant l'autonomie de gestion, les Directeurs disposent de marges de liberté importantes dans ce domaine; il en résulte qu'il n'y a pas uniformité au niveau de la gestion. Ainsi, alors que certaines caisses n'ont pas encore mis en place le contrôle de gestion, d'autres en ont développé des systèmes plus ou moins élaborés. D'où la question principale de cet article : quels sont les facteurs 
explicatifs de l'évolution, au sens d'émergence et de développement, du contrôle de gestion dans les CPAM?

Dans un premier temps, nous avons réalisé une enquête qualitative (1). Elle nous a permis d'identifier sur le terrain les facteurs qui a priori influaient sur l'évolution du contrôle de gestion dans les caisses (2). Nous avons pu ainsi proposer un modèle d'évolution du contrôle de gestion dans les CPAM (3). Un questionnaire a été élaboré (4) afin de collecter des données permettant de tester le modèle par le biais d'une analyse quantitative (5). Enfin, les résultats du test du modèle seront présentés de manière synthétique (6).

\section{L'enquête qualitative}

L'enquête qualitative qui s'est déroulée début 2000 a eu un caractère exploratoire du fait qu'aucune étude approfondie concernant le contrôle de gestion dans les CPAM n'avait encore été menée, ni sur le plan académique, ni par les différentes instances de l'Assurance maladie. Nous avons choisi de réaliser une étude multisites et non sur site unique car le contrôle de gestion présente des variétés dans la population des caisses et un site unique ne pouvait nous les faire découvrir. Une étude multisites nécessite de sélectionner un échantillon. Relativement à la méthodologie de mise en pratique de l'enquête qualitative, vont être exposés les choix concernant : le type d'échantillon (1.1); la méthodologie de constitution de l'échantillon (1.2); la conduite des entretiens et la technique d'exploitation des données (1.3).

\subsection{Le type d'échantillon : par homogénéisation}

Parmi les différentes possibilités de choisir un type d'échantillon dans le cadre d'une enquête qualitative, l'échantillonnage par homogénéisation a été adopté. Comme groupe homogène, la sous-population des CPAM pratiquant le contrôle de gestion a été retenue. Pour constituer l'échantillon, nous avons choisi des caisses les plus diverses possible en ce qui concerne la variable contrôle de gestion. Pour déterminer le nombre de caisses de l'échantillon (15, nombre relativement faible), a été utilisé le principe de saturation : des caisses supplémentaires n'auraient apporté qu'un gain marginal d'informations peu significatif.

\subsection{La méthodologie de constitution de l'échantillon : par filière}

Pour sélectionner les caisses, la technique de l'entretien par filière a été utilisée. Nous avons d'abord contacté l'une des CPAM les plus importantes de France. Son Agent comptable nous a aiguillé vers des CPAM pratiquant le contrôle de gestion, qui à leur tour, nous ont dirigé vers d'autres caisses pratiquant également le contrôle de gestion ou sur le point de le faire. Dans cet ensemble de caisses contactées, la diversification a été pratiquée. Afin de ne pas être éventuellement "prisonnier" d'un réseau de personnes se connaissant, des contacts téléphoniques "au hasard " ont aussi été réalisés qui nous ont mis en relation avec des CPAM n'envisageant pas de contrôle de gestion pour l'instant. Les caractéristiques des 15 caisses de l'échantillon sont présentées en annexe 1.

\subsection{La conduite des entretiens et la technique d'exploitation des données}

La technique de l'entretien non directif a été choisie. La majorité des entretiens ont eu lieu par téléphone, quatre en tête-à-tête. Les entretiens n'étaient pas enregistrés, l'information était consignée par prises de notes simultanées. Afin d'accroître la validité de l'enquête, les entretiens se sont caractérisés par une diversité des fonctions des répondants (voir annexe 1).

Notons le caractère confidentiel, tant au niveau de l'identification de la caisse que du répondant. Cette condition a été négociée avec les caisses avant le commencement de l'enquête.

Un guide d'entretien nous a aidé dans le déroulement des entretiens. Les thèmes suivants ont fait l'objet de questions : le contrôle de gestion, les autres systèmes d'information, les relations entre acteurs, les contraintes externes.

Concernant la technique d'exploitation des données de cette analyse qualitative, nous avons utilisé l'analyse thématique interentretiens. Les données recueillies ont fait l'objet d'un double traitement : d'une part, une analyse verticale concernant chaque caisse de l'échantillon et, d'autre part, une analyse horizontale concernant l'ensemble des caisses de l'échantillon.

\section{Les facteurs d'évolution du contrôle de gestion et la construction du modèle}

L'enquête qualitative nous a montré qu'il existe de nombreux facteurs conditionnant l'évolution du contrôle de gestion dans les CPAM. Nous les avons classés en trois types, en nous inspirant de la typologie d'Isabelle Danjou (1987) : les facteurs historiques (2.1); le facteur "chocs" (2.2); le facteur d'autonomie (2.3).

\subsection{Les facteurs historiques}

Les facteurs historiques résultent des choix du passé, tant au sein de la CPAM (facteurs internes) que de son environnement (facteurs externes). Ces facteurs ont été "construits" au cours du temps. Ils résultent d'une accumulation de faits, de décisions, d'événements, parfois sur des décennies. Certains de ces facteurs sont communs à l'ensemble des CPAM comme la contractualisation, d'autres sont, pour partie, spécifiques à 
chaque caisse comme la taille. À un instant donné, les facteurs historiques s'imposent à la CPAM, car ils sont invariants à court terme. Les facteurs historiques étant très nombreux, une sélection a été nécessaire afin que le modèle soit opérationnel. Les cinq facteurs suivants ont été retenus : le degré de développement du contrôle interne (2.1.1), la taille (2.1.2), la gestion d'œuvres (2.1.3), la contractualisation (2.1.4), la mise en place de nouvelles technologies (2.1.5). Nous pensons:

- qu'ils sont représentatifs d'une grande partie des autres facteurs historiques.

- que ce sont ceux qui influent le plus sur le contrôle de gestion. Nous avons abouti à cette conclusion grâce à l'enquête qualitative et à l'étude de différents modèles. Certains modèles d'évolution sont spécifiques au contrôle de gestion tels que ceux de R. Nolan (1974), D. Michel (1983), R.N. Anthony et D. Young (1988), S.M. Hronec (1995), R.S. Kaplan et D.P. Norton (1996). D'autres sont plus généraux tels que ceux de E. Penrose (1963), L.E. Greiner (1972), N. Churchill et V. Lewis (1983). Certains modèles de la contingence structurelle sont plus axés sur un facteur en particulier tel que la taille (D.S. Pugh et al, 1963; P.M. Blau, 1970; M. Basire, 1976; H. Mintzberg, 1982) ou la technologie (T.K. Reeves et J. Woodward, 1970; J. Cash et al, 1992).

\subsubsection{Le desré de développement du contrôle interne}

Une démarche de développement du contrôle interne est obligatoire dans les CPAM depuis 1993. Le degré de développement du contrôle interne est fonction de la culture, des jeux de pouvoir en place, de la logique de contractualisation et parfois de la taille. D'après une note interne de l'Assurance maladie en date du 22/11/1994, le contrôle interne "vise à protéger l'organisme contre les risques de toute nature entraînant un paiement injustifié ". La mise en place du contrôle interne incombe principalement à l'Agent comptable, mais le Directeur y est associé car ce type de contrôle implique l'organisation de la caisse qui est de son ressort. L'implantation du contrôle interne est un processus long. Notre étude qualitative nous a permis de constater que le niveau de développement du contrôle interne était très inégal suivant les CPAM. Notons que D. Michel (1983) considère le développement des procédures administratives, c'est-à-dire en fait du contrôle interne, comme un préalable au développement du contrôle de gestion.

\subsubsection{La taille en effectif (nombre de salariés)}

La taille en effectif influe très fortement sur la culture des CPAM, sur la présence syndicale et le jeu des acteurs. Nous pensons qu'elle est un indicateur pertinent de la complexité ${ }^{1}$

1. La complexité est fonction de nombreuses variables, telles que la taille, la difficulté des tâches, la multiplicité des publics, etc. (A. Burlaud et J.L. Malo, 1988). d'une CPAM. Les caisses ayant toutes globalement les mêmes missions, le même type de technologie, la principale variable qui permet d'apprécier leur différence au niveau de la complexité est la taille en effectif. Cette dernière est fonction des autres variables de taille, telles que le nombre d'actes à rembourser, le nombre d'immatriculations de nouveaux assujettis, le volume des paiements (sortie d'argent), etc. La taille reflète l'ampleur du travail à accomplir, c'est-à-dire l'intensité de l'activité à gérer. Les CPAM les plus "peuplées" en salariés sont théoriquement celles ayant à traiter le plus de travail.

\subsection{La gestion d'oeuvres}

Une œuvre est une organisation créée et gérée par une caisse primaire, telle qu'un centre de soins dentaires, un centre d'examen de santé, etc. La création d'œuvres et leur gestion est un choix et non une obligation pour chaque CPAM. Certaines œuvres sont gérées par des méthodes proches de celles du secteur privé et nous pensons qu'il y a une tendance à la diffusion de ces méthodes de gestion, dont le contrôle de gestion, dans le reste de la caisse. Ces œuvres, comme les centres de soins dentaires, doivent atteindre l'équilibre au niveau budgétaire sous peine, à terme, d'être éventuellement fermées ou de passer sous le contrôle d'un autre organisme que la CPAM.

La présence d'œuvres est notamment fonction de la volonté du Directeur, de la situation géographique, de la densité de population et de la situation sanitaire du département.

L'aspect "contagion" d'une technique ou d'une technologie au sens large mise en place dans un premier service puis ensuite étendue progressivement dans toute une organisation a notamment été développé dans la littérature par Cash J. et al (1992).

\subsubsection{La contractualisation}

La contractualisation s'impose à la population des CPAM. Elle résulte de choix gouvernementaux concernant l'ensemble du secteur public. La mise en place des contrats pluriannuels de gestion est l'aboutissement, au niveau local, c'est-à-dire des CPAM, de la logique de contractualisation. La contractualisation est indirectement à l'origine des systèmes de benchmarking mis en place dans l'Assurance maladie. Cette contractualisation tend à développer une culture de résultat.

De cette contractualisation découle deux logiques génératrices de contrôle de gestion :

- une logique interne : le contrôle de gestion permet aux caisses d'améliorer intrinsèquement leur gestion et donc d'être mieux classées les unes par rapport aux autres au niveau du benchmarking;

- une logique externe : la mise en place du contrôle de gestion, et surtout sa présence dans l'organigramme, peut être analysée comme un signal ${ }^{2}$ envoyé par les CPAM à la CNAMTS

2. Voir la théorie du signal in G. Charreaux (1993, p. 215), G. Hirigoyen et J.P. Jobard (1989, p. 1221-1241). 
pour pallier l'opacité informationnelle. Ce signal permet à la CNAMTS d'identifier les CPAM ayant décidé de transformer leurs méthodes de gestion pour accroître leur efficience. La CNAMTS leur alloue alors plus de ressources et leur attribue des avantages divers pour les récompenser de leurs efforts. Mentionnons ici la théorie de la dépendance des ressources de J. Pfeffer et G.R. Salanick (1978) qui résume l'environnement d'une organisation (ici une CPAM) à l'organisation qui lui permet d'acquérir des ressources (ici la CNAMTS).

\subsubsection{La mise en place des nouvelles technologies}

La technologie SÉSAM-VITALE (les télétransmissions) et l'informatisation des CPAM (le développement de l'Intranet, du groupware, etc.), modifient les conditions de travail dans les caisses. La mise en place des nouvelles technologies résulte de choix faits au niveau de la CNAMTS, même si chaque caisse possède une marge de manœuvre dans la vitesse de leur installation.

Au niveau d'une caisse, les télétransmissions entraînent de nécessaires réorganisations et nous pensons que cela déclenche un besoin de contrôle supplémentaire et, plus précisément, de contrôle de gestion. De plus, l'Intranet, les forums, la messagerie, le groupware facilitent l'assimilation du contrôle de gestion par les cadres (C. Bachelet et M.L. Caron, 1999) et sa diffusion dans la caisse.

Au niveau national, en reliant les caisses entre elles, ces technologies (Intranet, groupware, messagerie) favorisent les interactions et donc le développement du contrôle de gestion, par mimétisme, dans la population des caisses.

\subsection{Le facteur «chocs»}

Les chocs sont des événements non anticipés ou mal anticipés, extérieurs ou intérieurs à la CPAM, qui bousculent son équilibre en agissant directement sur elle ou en modifiant son environnement. Ils résultent souvent de facteurs historiques. Ils émergent plus ou moins soudainement d'un contexte; par exemple : une grève, les problèmes d'un nouveau système informatique, un changement soudain de la législation, etc. Ces chocs s'imposent aux CPAM qui n'ont pas de prise directe sur eux. En créant subitement du désordre, ils peuvent entraîner une réponse de la caisse et ainsi déclencher l'adoption du contrôle de gestion.

L'influence des chocs sur une organisation a notamment été étudiée par L.E. Greiner, (1972) qui montre qu'une entreprise évolue au cours de sa vie par crises successives. Pour R. Cyert et J. March (1963), les éléments déclenchant l'apprentissage d'une organisation sont les chocs externes qui perturbent le système organisationnel. R.N. Anthony et D. Young (1988) mentionnent le rôle de chocs sur le contrôle de gestion dans les "non-profit organisations" dont les CPAM font d'ailleurs partie.

Les facteurs historiques et les chocs sont déterministes. La CPAM n'a pas le choix, elle ne peut, à court terme, se dégager de leur influence. Notons que les facteurs historiques sont prévisibles alors que les chocs le sont beaucoup moins, voire pas du tout. En sus de ces facteurs déterministes, le modèle intègre un facteur de liberté de l'acteur que nous appellerons le facteur d'autonomie.

\subsection{Le facteur d'autonomie : la volonté du Directeur}

L'émergence et le développement du contrôle de gestion ne se font pas mécaniquement en fonction des facteurs historiques et des chocs, mais résultent également d'un choix.

L'aspect non déterministe de l'évolution a été développé par des auteurs tels que E. Penrose (1963), N. Churchill et V. Lewis (1983). Plus spécifiquement, les modèles issus du nouveau contrôle de gestion (S.M. Hronec, 1995; R.S. Kaplan et D.P. Norton 1996) mettent l'accent sur l'importance de la volonté dans la mise en place du contrôle de gestion

Nous réduisons ce facteur d'autonomie à la volonté d'un seul acteur: le Directeur. En effet, c'est le Directeur qui prend la décision d'implantation du contrôle de gestion, car il a en charge l'organisation de la CPAM. Sa liberté est néanmoins conditionnée tant par les facteurs historiques et les chocs que par l'influence d'autres acteurs tels que l'encadrement, l'Agent comptable, la CNAMTS etc. Ces différents acteurs peuvent agir en sens opposés, certains freinant le contrôle de gestion, d'autres incitant le Directeur à l'adopter. Le contrôle de gestion, en introduisant plus de transparence, réduit la marge d'incertitude de l'encadrement. L'introduction du contrôle de gestion va déranger l'équilibre des pouvoirs en place, ce qui peut donc être source de résistance de la part de certains acteurs. Ce type de problématique a été développé, de façon générale, par M. Crozier et E. Friedberg (1977) et E. Friedberg (1993).

Le facteur "volonté du Directeur" est primordial. En effet, sans cette volonté, quels que soient les facteurs historiques et l'importance des chocs, le contrôle de gestion restera lettre morte.

\section{Modèle d'évolution du contrôle de gestion dans les CPAM}

Les différentes hypothèses du modèle d'évolution retenues sont présentées dans le tableau 1.

Afin de tester le modèle, nous avons réalisé une étude quantitative à destination de l'ensemble des CPAM. Cette étude quantitative a été menée par le biais d'un questionnaire postal.

Tableau 1. Les hypothèses du modèle

\begin{tabular}{|l|l|}
\hline Hypothèse & \multicolumn{1}{c|}{ Énoncé de l'hypothèse (la proposition) } \\
\hline H1 & $\begin{array}{l}\text { La présence du contrôle interne est un facteur } \\
\text { favorable à l'émergence et au développement du } \\
\text { contrôle de gestion. }\end{array}$ \\
\hline
\end{tabular}




\begin{tabular}{|c|c|}
\hline Hypothèse & Énoncé de l'hypothèse (la proposition) \\
\hline $\mathrm{H} 2$ & $\begin{array}{l}\text { La gestion d'œuvres par une CPAM est un facteur } \\
\text { favorable à l'émergence et au développement du } \\
\text { contrôle de gestion }\end{array}$ \\
\hline H3 & $\begin{array}{l}\text { Plus la taille (en effectif) d'une CPAM est importante, } \\
\text { plus le contrôle de gestion est susceptible d'émerger } \\
\text { et de se développer. }\end{array}$ \\
\hline $\mathrm{H} 4$ & $\begin{array}{l}\text { Les nouvelles technologies ont une influence favorable } \\
\text { sur l'émergence et le développement du contrôle de } \\
\text { gestion. }\end{array}$ \\
\hline H4.1 & $\begin{array}{l}\text { L'Intranet et les différentes sources d'interaction qui } \\
\text { en découlent (groupware, forum, etc.) sont un élément } \\
\text { favorable à la diffusion du contrôle de gestion au sein } \\
\text { de chaque CPAM et dans la population des CPAM. }\end{array}$ \\
\hline $\mathrm{H} 4.2$ & $\begin{array}{l}\text { La technologie de la carte SÉSAM-VITALE (télétrans- } \\
\text { missions) est un élément favorable au développement } \\
\text { du contrôle de gestion au sein d'une CPAM. }\end{array}$ \\
\hline H5 & $\begin{array}{l}\text { Les chocs ont une influence favorable sur l'émergence } \\
\text { et le développement du contrôle de gestion. }\end{array}$ \\
\hline H6 & $\begin{array}{l}\text { Le contrôle de gestion émerge et se développe dans } \\
\text { les CPAM suite à une modification de l'environnement } \\
\text { résultant de la mise en place de la contractualisation } \\
\text { dans l'Assurance maladie. }\end{array}$ \\
\hline $\mathrm{H} 7$ & $\begin{array}{l}\text { Il existe une logique interne à l'émergence et au } \\
\text { développement du contrôle de gestion dans une } \\
\text { CPAM : il lui permet d'améliorer sa gestion. }\end{array}$ \\
\hline H8 & $\begin{array}{l}\text { Il existe une logique externe à l'émergence et au } \\
\text { développement du contrôle de gestion dans une } \\
\text { CPAM : il est un signal de bonne gestion à destination } \\
\text { de la CNAMTS et des autres acteurs extérieurs. }\end{array}$ \\
\hline H9 & $\begin{array}{l}\text { L'implantation (émergence et développement) du } \\
\text { contrôle de gestion résulte de la volonté du Directeur. }\end{array}$ \\
\hline
\end{tabular}

\section{Le questionnaire de l'enquête quantitative : destination, présentation et administration}

Le questionnaire comporte 105 questions. Vu diverses bifurcations, les répondants n'avaient en fait à répondre qu'à environ 80 questions en cas de présence du contrôle de gestion, et à environ 40 en cas d'absence du contrôle de gestion. La majeure partie du questionnaire est constitué de questions fermées. Les plus simples de nos questions fermées sont de type booléen (oui/non). D'autres questions proposent plus de deux items avec un seul choix possible ou plusieurs choix possibles (questions à choix multiples). Certaines sont des questions avec échelle d'attitude (de type Likert). D'autres demandent au répondant de classer les items les uns par rapport aux autres. Certaines questions fermées ont été accompagnées d'une possibilité de commentaires qualitatifs.

\subsection{La destination du questionnaire}

Le questionnaire a été envoyé, par voie postale, en novembre 2000 aux 128 CPAM de France métropolitaine. L'enquête avait vocation à être exhaustive (recensement). Le questionnaire a été adressé au Directeur de chaque caisse. En effet, l'organisation de la CPAM étant du ressort de ce dernier, il a seul la possibilité de proposer la mise en place d'un système de contrôle de gestion. Nous avons néanmoins précisé dans la lettre d'introduction au questionnaire que le Directeur pouvait déléguer, en tout ou en partie, la réponse au questionnaire à l'Agent comptable ou à tout autre agent.

\subsection{La présentation et l'administration du questionnaire}

Le questionnaire comportait trois parties qui sont, dans l'ordre, l'organisation de la caisse, le contrôle interne et le contrôle de gestion. Avant d'être diffusé dans l'ensemble des CPAM, le questionnaire a été testé par des acteurs du milieu concerné et par des répondants potentiels, tant au niveau de sa construction que de la formulation de ses questions.

Le retour des questionnaires a pris bien plus de temps que prévu. Nous pensions qu'il s'étalerait sur 3 mois et il s'est étendu sur 18 mois (relances comprises). En effet, le questionnaire a souvent circulé entre différents acteurs de la caisse, en liaison avec les différents thèmes qu'il comporte; de plus, de nombreux questionnaires (plus d'une vingtaine) ont été égarés dans les CPAM, ce qui a nécessité des renvois.

\section{L'analyse quantitative}

La principale source de données de l'analyse quantitative provient du dépouillement du questionnaire. Le taux de réponses a été de 87,5\%, c'est à dire de 112 questionnaires exploitables. L'analyse des 16 CPAM non répondantes ne fait pas apparaître de biais systématique significatif. De ce fait, l'enquête quantitative présente une validité externe restreinte satisfaisante (extrapolation des résultats de l'étude à la population totale des CPAM). Les données du questionnaire ont été complétées par des données quantitatives obtenues par le biais de la CNAMTS et de diverses sources, notamment des documents joints aux questionnaires par les répondants.

Les données ont été saisies et dépouillées sur le logiciel ÉTHNOS. En logiciels complémentaires, EXCEL et SPAD ont été utilisés. Ont été réalisées des analyses univariée, bivariée (5.1) et multivariée (5.2).

\subsection{L'analyse univariée et l'analyse bivariée}

Seront étudiés successivement les liens suivants découlant des hypothèses du modèle : contrôle de gestion/contrôle interne (5.1.1); contrôle de gestion/gestion d'œuvres (5.1.2); contrôle de gestion/taille (5.1.3); contrôle de gestion/Intranet (5.1.4); contrôle de gestion/télétransmissions (5.1.5); contrôle de gestion/chocs 
(5.1.6); contrôle de gestion/contractualisation (5.1.7); contrôle de gestion/logique interne (5.1.8); contrôle de gestion/logique externe (5.1.9); contrôle de gestion/Directeur (5.1.10).

\subsubsection{Le lien contrôle de gestion/contrôle interne (hypothèse 1)}

Au niveau des caisses ayant répondu, 41,96 \% (47/112) ont un service en charge du contrôle de gestion. Afin de tester l'hypothèse H1, dans le tableau 2 a été croisée la présence du contrôle de gestion avec le niveau de développement du contrôle interne.

Tableau 2. Croisement présence du contrôle de gestion/niveau de développement du contrôle interne

\begin{tabular}{|l|c|c|c|}
\cline { 2 - 4 } \multicolumn{1}{c|}{} & $\begin{array}{c}\text { Contrôle } \\
\text { de gestion }\end{array}$ & $\begin{array}{c}\text { Sans contrôle } \\
\text { de gestion }\end{array}$ & $\begin{array}{c}\text { Total } \\
\text { des CPAM }\end{array}$ \\
\hline $\begin{array}{l}\text { Cl bien } \\
\text { développé }\end{array}$ & 16 & 11 & 27 \\
\hline $\begin{array}{l}\text { Cl assez bien } \\
\text { développé }\end{array}$ & 18 & 23 & 41 \\
\hline $\begin{array}{l}\text { Cl peu } \\
\text { développé }\end{array}$ & 13 & 31 & 44 \\
\hline Total des CPAM & 47 & 65 & 112 \\
\hline
\end{tabular}

D'après le test du Chi2 (Chi2 calculé $=6,17$, Chi2 ${ }_{0,05}=5,99$, 2 ddl), le niveau de développement du contrôle interne et la présence de contrôle de gestion sont liés. En d'autres termes, le contrôle de gestion est d'autant plus présent que le contrôle interne est développé.

Le dépouillement du questionnaire a également permis de constater que 46,66 \% des caisses (avec contrôle de gestion) pensent que la mise en place de leur contrôle de gestion a été facilitée par l'implantation préalable du contrôle interne. De plus, pour 51,28 \% des répondants, le niveau de développement du contrôle interne détermine la vitesse de développement du contrôle de gestion.

Malgré les différents résultats précédents, nous considérons que I'hypothèse $\mathrm{H} 1$ n'est validée qu'en partie seulement. En effet, la présence de contrôle interne est bien un élément favorable à l'émergence et au développement du contrôle de gestion. Mais, le lien niveau de développement du contrôle interne/niveau de développement du contrôle de gestion n'a pas été testé (vu le taux important de non-réponses concernant le niveau de développement du contrôle de gestion). C'est pour cela que nous optons pour une validation partielle. Ce lien nécessite un approfondissement et pourra faire l'objet d'une recherche ultérieure.

\subsubsection{Le lien contrôle de gestion/gestion d'oeuvres (hypothèse 2)}

Les caisses gèrent des œuvres dans 48,21 \% des cas (54/112 $=48,21 \%$ ), et principalement des centres d'examen de santé et des centres de soins dentaires. D'autres types de centres sont présents en petit nombre (dispensaire, centre de Protection Maternelle et Infantile, centre de réadaptation fonctionnelle). L'on peut noter que seuls les centres de soins dentaires ont une gestion qui se rapproche de celle du secteur privé avec des ressources propres (œuvres que nous qualifierons de privées). Pour les autres centres, l'équilibre comptable est uniquement atteint grâce à des subventions (œuvres que nous qualifierons de publiques).

Deux tableaux croisés synthétiques ont d'abord été réalisés. Le premier (tableau 3) croise la présence du contrôle de gestion et la présence d'œuvres de tous types dans les CPAM.

Tableau 3. Croisement Présence du contrôle de gestion/OEuvres de tous types

\begin{tabular}{|l|c|c|c|}
\cline { 2 - 4 } \multicolumn{1}{c|}{} & $\begin{array}{c}\text { Contrôle } \\
\text { de gestion }\end{array}$ & $\begin{array}{c}\text { Sans contrôle } \\
\text { de gestion }\end{array}$ & $\begin{array}{c}\text { Total } \\
\text { des CPAM }\end{array}$ \\
\hline CEuvres & 27 & 27 & 54 \\
\hline Sans œuvres & 20 & 37 & 58 \\
\hline $\begin{array}{l}\text { Total des } \\
\text { CPAM }\end{array}$ & 47 & 65 & 112 \\
\hline
\end{tabular}

D'après le test du Chi2 (Chi2 calculé, avec la correction de Yates $=1,97$, Chi2 ${ }_{0,10}=2,71,1 \mathrm{ddl}$ ), on ne peut conclure sur le lien entre la présence d'œuvres et la présence du contrôle de gestion. Le deuxième (tableau 4) croise la présence du contrôle de gestion et la présence d'œuvres privées.

Tableau 4. Croisement Présence du contrôle de gestion/OEuvres privées

\begin{tabular}{|l|c|c|c|}
\cline { 2 - 4 } \multicolumn{1}{c|}{} & $\begin{array}{c}\text { Contrôle } \\
\text { de gestion }\end{array}$ & $\begin{array}{c}\text { Sans contrôle } \\
\text { de gestion }\end{array}$ & $\begin{array}{c}\text { Total } \\
\text { des CPAM }\end{array}$ \\
\hline $\begin{array}{l}\text { CEuvres privées } \\
\text { (centres de soins } \\
\text { dentaires) }\end{array}$ & 16 & 9 & 25 \\
\hline Sans œuvres privées & 31 & 56 & 87 \\
\hline Total des CPAM & 47 & 65 & 112 \\
\hline
\end{tabular}

D'après le test du Chi2 (Chi2 calculé, avec la correction de Yates $=5,31$, Chi2 ${ }_{0,025}=5,02,1 \mathrm{ddl}$ ), les œuvres ayant une gestion de type privé et le contrôle de gestion sont liés.

L'hypothèse $\mathrm{H} 2$ est validée pour les œuvres privées, mais pas pour les œuvres en général.

\subsubsection{Le lien contrôle de sestion/taille (hypothèse 3)}

La population des 112 caisses "répondantes" se scinde en deux sous-populations de caisses, respectivement avec et sans contrôle de gestion. La comparaison directe des paramètres de taille de ces deux sous-populations (tableau 5) est significative du fait que les 112 caisses répondantes constituent pratiquement la population totale des caisses (taux de réponse : 112/128 $=87,5 \%)$.

On constate (tableau 5) que l'effectif moyen des caisses avec contrôle de gestion est clairement supérieur à celui des caisses 
sans contrôle de gestion. Le lien contrôle de gestion/taille est donc très net. Notons que les dispersions relatives (coefficients de dispersion) sont pratiquement égales.

Afin de corroborer le lien contrôle de gestion/taille, nous avons élaboré le tableau 6 dans lequel la variable quantitative "effectif " a été muée en variable qualitative.

Tableau 5. Présence du contrôle de gestion et statistiques concernant les effectifs

\begin{tabular}{|l|c|c|}
\cline { 2 - 3 } \multicolumn{1}{c|}{} & $\begin{array}{c}\text { Contrôle } \\
\text { de gestion }\end{array}$ & $\begin{array}{c}\text { Sans contrôle } \\
\text { de gestion }\end{array}$ \\
\hline Nombre de CPAM répondantes & 47 & 65 \\
\hline $\begin{array}{l}\text { Total des effectifs des CPAM } \\
\text { (nombre de salariés) }\end{array}$ & 30346 & 26529 \\
\hline $\begin{array}{l}\text { Moyenne des effectifs } \\
\text { des caisses }\end{array}$ & 645,66 & 408,14 \\
\hline $\begin{array}{l}\text { Écart type des effectifs } \\
\text { des caisses }\end{array}$ & 644,35 & 386,06 \\
\hline $\begin{array}{l}\text { Coefficient de dispersion } \\
\text { (Écart type/Moyenne) }\end{array}$ & 1,00 & 0,95 \\
\hline
\end{tabular}

Tableau 6. Croisement Présence du contrôle de sestion et Taille en effectif

\begin{tabular}{|l|l|l|l|}
\cline { 2 - 4 } \multicolumn{1}{c|}{} & \multicolumn{1}{c|}{$\begin{array}{c}\text { Contrôle } \\
\text { de gestion }\end{array}$} & \multicolumn{1}{c|}{$\begin{array}{c}\text { Sans contrôle } \\
\text { de gestion }\end{array}$} & $\begin{array}{c}\text { Total } \\
\text { des CPAM }\end{array}$ \\
\hline $\begin{array}{l}\text { Très petite } \\
\text { (de 80 à 250) }\end{array}$ & 10 & 22 & 32 \\
\hline $\begin{array}{l}\text { Petite } \\
\text { (de 250 à 500) }\end{array}$ & 20 & 29 & 49 \\
\hline $\begin{array}{l}\text { Moyenne } \\
\text { (de 500 à 800) }\end{array}$ & 6 & 11 & 17 \\
\hline $\begin{array}{l}\text { Importante } \\
\text { (de 800 à 3500) }\end{array}$ & 11 & 3 & 14 \\
\hline Total des CPAM & 47 & 65 & 112 \\
\hline
\end{tabular}

D'après le test du Chi2 (Chi2 calculé $=9,55$, Chi2 ${ }_{0,025}=9,35$, 3 ddl), la présence du contrôle de gestion et la taille sont liés.

$\mathrm{Au}$ vu de ces différents résultats, il ressort que l'hypothèse H3 est validée.

\subsubsection{Le lien contrôle de gestion/Intranet (hypothèse H4.1)}

L'influence, sur le contrôle de gestion, de la présence d'un intranet (hypothèse $\mathrm{H} 4.1$ ) est la première composante de l'hypothèse H4. On constate que 57,45 \% (27/47) des caisses pratiquant le contrôle de gestion ont mis en place un Intranet.

Dans $61,54 \%$ des cas, elles s'en servent pour diffuser des productions du contrôle de gestion (indicateurs, tableaux de bord, objectifs).

De plus, pour 50 \% des caisses pratiquant le contrôle de gestion mais n'ayant pas mis en place un réseau Intranet, I'utilisation d'un Intranet aurait un effet positif sur le contrôle de gestion, notamment en accélérant son développement. Nous en concluons que I'hypothèse H4.1 est validée.

\subsubsection{Le lien contrôle de gestion/télétransmissions (hypothèse H4.2)}

L'influence, sur le contrôle de gestion, du développement des télétransmissions (hypothèse H4.2), est la deuxième composante de l'hypothèse $\mathrm{H} 4$.

Pour seulement 36,36 \% des caisses pratiquant le contrôle de gestion, la mise en place des télétransmissions (SÉSAM-VITALE) favorise le contrôle de gestion. Mais cela est vrai pour 59,32 \% des caisses sans contrôle de gestion. Ce pourcentage est supérieur à celui des caisses avec contrôle de gestion (36,36 \%), car ces dernières ont peut-être constaté que l'utilité du contrôle de gestion se situe essentiellement ailleurs que dans le domaine de la réorganisation. De ce fait, nous considérons que l'hypothèse H4.2 n'est que partiellement validée.

L'hypothèse H4.1 étant validée mais I'hypothèse H4.2 n'étant que partiellement validée, I'hypothèse $\mathrm{H} 4$ dans sa globalité est partiellement validée.

\subsubsection{Le lien contrôle de gestion/chocs (hypothèse 5)}

D'après les réponses à notre questionnaire, seules deux CPAM ont mis en place le contrôle de gestion suite à un choc. Les chocs ne sont donc pas un facteur déterminant dans la mise en place du contrôle de gestion.

La mise en place du contrôle de gestion comme moyen préventif pour faire face à un choc futur n'apparaît pas non plus comme une raison première tant pour les caisses pratiquant le contrôle de gestion que pour les caisses ne le pratiquant pas.

Concernant l'influence des chocs sur le développement du contrôle de gestion, seulement dans un cas, le contrôle de gestion a vu son développement accéléré par un choc. Dans ce cas précis, le choc était une grève.

Vu la faiblesse de l'influence favorable des chocs sur l'émergence et le développement du contrôle de gestion, l'hypothèse H5 n'est pas validée. Notre enquête par questionnaire infirme donc ici l'intuition que nous avions eue lors de l'enquête qualitative et par l'analyse de la littérature.

\subsubsection{Le lien contrôle de gestion/contractualisation (hypothèse 6)}

Le lien contractualisation/contrôle de gestion est manifeste. En effet, $85,58 \%$ des répondants considèrent que la contractualisation des relations entre la CNAMTS et les CPAM (les contrats pluriannuels de gestion) favorise l'émergence du contrôle de gestion.

Cela confirme notre intuition que le besoin de contrôle de gestion est, entre autres, né de la modification de l'environnement et plus particulièrement d'une réforme venue du sommet de l'État. L'hypothèse $\mathrm{H} 6$ est ainsi validée. 
Le rôle de la contractualisation va être approfondi. En effet, de la contractualisation découlent deux logiques d'introduction du contrôle de gestion : la logique interne et la logique externe.

\subsubsection{Le lien contrôle de gestion/logique interne (hypothèse 7)}

Le contrôle de gestion est un moyen d'améliorer sa gestion (et donc son classement au niveau du benchmarking) pour 65,22 \% des répondants l'ayant mis en place et pour 58,62 \% des répondants ne l'ayant pas mis en place.

De plus, pour $84,43 \%$ des répondants l'ayant mis en place et pour $91,53 \%$ des répondants ne l'ayant pas mis en place, la raréfaction relative des ressources de gestion administrative favorise l'émergence du contrôle de gestion. Cela montre que le contrôle de gestion est considéré comme un outil permettant d'accroître l'efficience dans les caisses.

Du fait des résultats précédents, nous considérons que l'hypothèse $\mathrm{H} 7$ est validée.

\subsubsection{Le lien contrôle de gestion/logique externe (hypothèse 8)}

Concernant les CPAM faisant du contrôle de gestion, a été étudiée l'influence, sur l'attitude de la CNAMTS, de la présence du contrôle de gestion, puis du niveau de développement du contrôle de gestion. Pour 65,91 \% des répondants, le fait de pratiquer le contrôle de gestion est un atout vis-à-vis de la CNAMTS car cela permet d'obtenir plus de ressources de sa part lors de la négociation des contrats pluriannuels de gestion. Pour $71,43 \%$ des répondants, le niveau de développement du contrôle de gestion n'est pas considéré comme important. Tout se passe comme si seul comptait l'effet d'affichage, dans l'organigramme, d'un service de contrôle de gestion.

Concernant les CPAM ne faisant pas de contrôle de gestion, pour seulement 45,9 \% des répondants, la pratique du contrôle de gestion serait un atout vis-à-vis de la CNAMTS car cela permettrait d'obtenir de sa part plus de ressources lors de la négociation des contrats pluriannuels de gestion. Ce pourcentage est beaucoup plus faible que celui des caisses pratiquant le contrôle de gestion $(65,91 \%)$. Nous pouvons ainsi penser que l'effet de signal n'est pas le but premier dans la mise en place du contrôle de gestion. Mais une fois ce dernier implanté, l'effet de signal se révèle et devient une motivation pour conserver le contrôle de gestion. Nous considérons que l'hypothèse $\mathrm{H} 8$ est partiellement validée.

\subsubsection{Le lien contrôle de gestion/volonté du Directeur (hypothèse 9)}

Pour près de $85 \%$ des répondants pratiquant le contrôle de gestion, sa mise en place est le fait principalement de la décision du Directeur. Pour les 15 \% restants, sont cités comme principaux acteurs de la mise en place, l'Agent Comptable et, dans une moindre mesure le Conseil d'Administration. L'hypothèse H9 est donc validée.

\subsection{L'analyse multivariée}

Précédemment, des analyses bivariées ont étudié les liens entre les variables prises deux à deux.

Afin d'obtenir une vue globale, une méthode d'analyse des données a été appliquée à une partie des variables du modèle. Seront exposés, la méthode de travail (5.2.1), puis les résultats et leur interprétation (5.2.2).

\subsubsection{La méthode de travail}

L'Analyse Factorielle des Correspondances (AFC) et son extension, I'Analyse Factorielle des Correspondances Multiples (AFCM), sont des instruments privilégiés pour le traitement des enquêtes par questionnaire comportant des questions fermées et dont les variables sont qualitatives. Pour réaliser l'AFCM, quatre variables ont été retenues : le degré de développement du contrôle interne; la présence d'œuvres; la taille en effectif (nombre de personnes travaillant dans la caisse); la présence de contrôle de gestion. Les variables suivantes ont été exclues : d'une part, le facteur chocs (H5) car non validé par l'analyse bivariée et, d'autre part, les nouvelles technologies ( $\mathrm{H} 4$ qui englobe $\mathrm{H} 4.1$ et $\mathrm{H} 4.2$ ), la contractualisation $(\mathrm{H} 6)$, la logique interne $(\mathrm{H} 7)$, la logique externe (H8) car non spécifiques à une caisse en particulier mais concernant la population d'organisations dans son ensemble (donc sans pouvoir discriminant). Dans le tableau 7, sont présentées les modalités des variables utilisées dans I'AFCM avec leur dénomination dans le logiciel SPAD.

Contrôle interne, œuvres et taille sont des variables explicatives du contrôle de gestion dans le modèle. L'AFCM va mettre en relief les proximités des modalités des variables et les visualiser sous forme de graphique. L'analyse s'intéressera aux axes 1et 2, les plus explicatifs pour notre problématique. En effet, ces deux axes ont les inerties les plus élevées (respectivement 19,67\% et $15,04 \%$ ). Voir l'annexe 2 pour le détail des différents paramètres chiffrés de l'AFCM.

\subsubsection{Les résultats de l'AFCM et leur interprétation}

La figure 1 situe les modalités des variables dans le plan F1 F2. Les deux axes F1 et F2 corroborent les résultats de l'analyse bivariée. La figure 1 montre une proximité entre CTG, CEuvres privées, Cl Bien développé, Importante (taille). Les caisses pratiquant le contrôle de gestion ont donc tendance à posséder ces différentes caractéristiques, c'est à dire à gérer des œuvres de type privé, à pratiquer le contrôle interne et à être de grande taille. 
Tableau 7. Les modalités des variables utilisées dans I'AFCM

\begin{tabular}{|c|c|c|c|}
\hline Variables & Nombre de modalités pour chaque variable & Modalités telles qu'elles apparaissent sur les graphiques & Effectifs par modalité \\
\hline $\begin{array}{l}\text { Le degré de dévelop- } \\
\text { pement du contrôle } \\
\text { interne }\end{array}$ & 4 & $\begin{array}{l}\text { Cl Pratiquement pas développé } \\
\text { Cl Peu développé } \\
\text { Cl Assez bien développé } \\
\text { Cl Bien développé } \\
\text { Les modalités "Cl Bien développé" (24) et "Cl Très } \\
\text { bien développé" (3) ont été regroupées. } \\
\end{array}$ & $\begin{array}{l}7 \\
37 \\
41 \\
27\end{array}$ \\
\hline Les œuvres & 3 & $\begin{array}{l}\text { Pas d'œuvre } \\
\text { CEuvres privées } \\
\text { CEuvres publiques }\end{array}$ & $\begin{array}{l}58 \\
25 \\
29\end{array}$ \\
\hline La taille & 4 & $\begin{array}{l}\text { Très petite } \\
\text { Petite } \\
\text { Moyenne } \\
\text { Importante } \\
\text { Les modalités "Très grande" (2 caisses) "Grande" } \\
\text { (4) et "Assez grande" (8) ont été regroupées dans la } \\
\text { modalité "Importante". }\end{array}$ & $\begin{array}{l}32 \\
49 \\
17 \\
14\end{array}$ \\
\hline $\begin{array}{l}\text { La présence de } \\
\text { contrôle de gestion }\end{array}$ & 2 & $\begin{array}{l}\text { PAS DE CTG } \\
\text { CTG (c'est-à-dire présence de contrôle de gestion) }\end{array}$ & $\begin{array}{l}65 \\
47\end{array}$ \\
\hline
\end{tabular}

Tableau 8. Synthèse des résultats du test du modèle

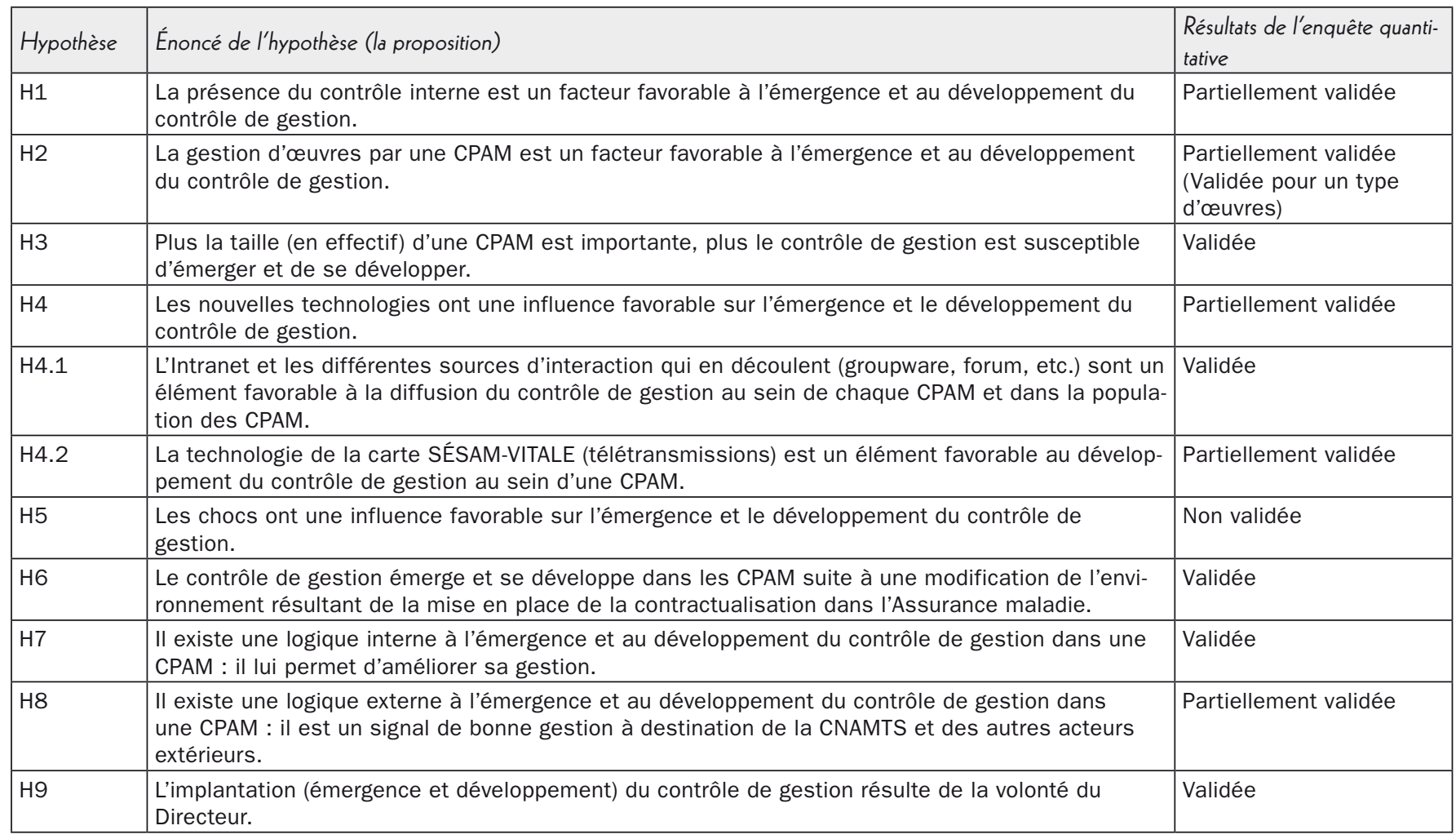


Facteur $2 \cdot 15.04 \%$

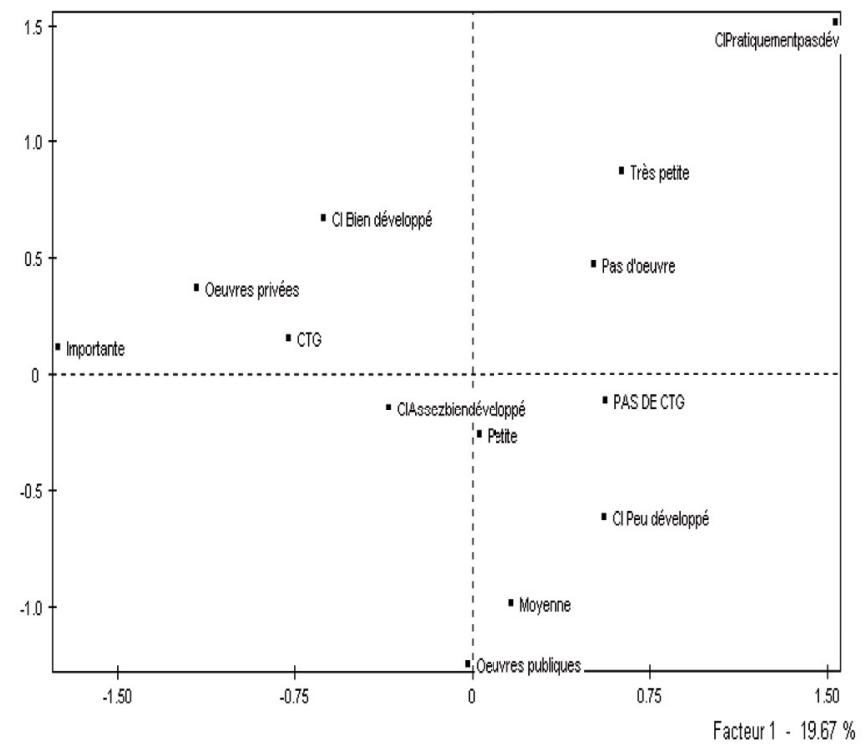

Figure 1. La représentation des modalités des variables dans le plan F1/F2 (SPAD)

\section{Les résultats, sous forme synthétique, du test du modèle}

Après application des analyses univariée, bivariée et multivariée, I'on aboutit aux résultats suivants concernant les hypothèses du modèle :

\section{Conclusion}

Après avoir réalisé une enquête qualitative concernant le contrôle de gestion dans quinze caisses, nous avons proposé un modèle d'évolution de ce système d'information dans le milieu spécifique des CPAM. Ce modèle a ensuite été testé par une enquête quantitative et globalement validé.

Le modèle pourrait être extrapolé, moyennant éventuellement certains aménagements, à toute organisation ayant une mission de service public, une autonomie de gestion et faisant partie d'une population d'organisations similaires structurées en réseau. Ainsi, il pourrait être transposé dans les secteurs suivants :

- aux autres branches de la Sécurité sociale du Régime général (branche vieillesse, branche famille); ainsi, les CAF (Caisses d'Allocations Familiales) ont une organisation très proche des CPAM ;

- à certaines mutuelles, notamment celles qui travaillent déjà avec les CPAM, comme la MGEN (Mutuelle Générale de l'Éducation Nationale);

- aux systèmes étrangers de sécurité sociale laissant une large place à l'autonomie, tout en étant de service public ou corporatiste;
- à une structure de type Pôle Emploi, anciennement ANPE (Agence Nationale Pour l'Emploi) et Assedic (Association pour l'Emploi dans l'Industrie et le Commerce).

\section{Bibliographie}

Anthony R.N. (1988), "The Management Control Fonction ", The Harvard Business Press.

Anthony R.N. et Young D. (1988), "Management Control in Non-Profit Organizations ", Irwin.

Bachelet C. et Caron M.L. (1999), "Groupware et impacts organisationnels ", Congrès de l'AGRH, Lyon, septembre.

Basire M. (1976), "La théorie des cinq niveaux", Direction et Gestion, $n^{\circ} 3$, 4 et 5 .

Blau P.M. (1970), "The Formal Theory of Differentiation", American Sociology Review, vol 35, p. 201-218.

Bouquin H. (1997), "Le contrôle de Gestion ", PUF.

Burlaud A. et Malo J.L. (1988), "Les organisations complexes : un défi aux méthodes traditionnelles de contrôle de gestion ", Revue Française de Comptabilité, février.

Cash J., Mac Kenney J., Applegate L. (1992), "Corporate Information System Management", Irwin.

Casin P. (1999), "Analyse des données et des panels de données", De Boeck Université.

Charreaux G. (1993), "Gestion financière ", Litec.

Churchill N. et Lewis V. (1983), "Les cinq stades de l'évolution d'une PME ", Harvard l'expansion, $n^{\circ} 30$.

Crozier M., Friedberg E. (1977), "L'acteur et le système", Le Seuil.

Cyert R. et March J. (1963), "A Behavioral Theory of The Firm ", Prentice Hall, Englewood Cliffs.

Danjou I. (1987), "L'évolution de la firme: analyse des facteurs et processus d'évolution à l'aide d'une approche monographique ", Thèse en Sciences de Gestion, Lille.

Friedberg E. (1993), "Le pouvoir et la règle: dynamique de l'action organisée", Les Editions du Seuil.

Greiner L.E. (1972), "Evolution and Revolution as Organizations Grow ", Harvard Business Review, juillet-août.

Hirigoyen G. et Jobard J.P. (1989), " Financement de l'entreprise : évolution récente et perspectives nouvelles" in Encyclopédie de gestion, Économica, volume 2, p. 1221-1241.

Hronec S.M. (1995), "Vital signs", Les Editions d'Organisation.

Kaplan R.S. et Norton D.P. (1996), "The Balance Scorecard", Harvard Business School Press.

Michel D. (1983), "Les systèmes de contrôle de gestion ", Les cahiers français, $n^{\circ} 210$, mars-avril.

Mintzberg H. (1982), "Structure et dynamique des organisations ", Les Editions d'Organisation.

Nolan R. (1974), "Managing the Four Stages of EDP Growth ", Harvard Business Review, janvier-février.

Penrose E. (1963), "Facteurs, conditions et mécanismes de la croissance de l'entreprise", Hommes et techniques.

Pfeffer J et G. R Salanick (1978), "The External Control of Organizations, a Ressource Dependance Perspective", New York, Harper and Row.

Pugh D.S., Hickson D.J. et al (1963), "A Conceptual Scheme for Organizational Analysis ", Administrative Science Quaterly, Vol 8, p. 289-315.

Reeves T.K. et Woodward J. (1970), «Industrial Organization : Behavior and Control ", Oxford University Press. 


\section{Annexes}

\section{Annexe 1}

Tableau 9. Récapitulatif des caractéristiques des caisses de l'enquête qualitative.

\begin{tabular}{|c|c|c|c|c|c|c|c|}
\hline Caisse & Région & $\begin{array}{l}\text { Taille arrondie de } \\
\text { la caisse en effectif }\end{array}$ & $\begin{array}{l}\text { Nombre de } \\
\text { répondants }\end{array}$ & Fonction du ou des répondants & $\begin{array}{c}\text { Nombre total } \\
\text { d'entretiens }\end{array}$ & $\begin{array}{c}\text { Durée du ou des } \\
\text { entretiens }\end{array}$ & $\begin{array}{c}\text { Étalement } \\
\text { dans le temps }\end{array}$ \\
\hline 1 & Lorraine & 440 & 3 & $\begin{array}{l}\text { Auditeur Interne } \\
\text { Contrôleur interne } \\
\text { Sous-directeur }\end{array}$ & 5 & $\begin{array}{l}\text { Deux fois une } \\
\text { heure } \\
\text { Deux fois une } \\
\text { heure } \\
\text { Trois heures }\end{array}$ & $\begin{array}{l}\text { Un an et } \\
\text { demi }\end{array}$ \\
\hline 2 & Ile-de-France & 1720 & 1 & Contrôleur de gestion & 3 & Une heure chacun & 3 mois \\
\hline 4 & Lorraine & 630 & 1 & Contrôleur de gestion & 1 & $\begin{array}{l}\text { Trois-quarts } \\
\text { d'heure }\end{array}$ & - \\
\hline 5 & Limousin & 370 & 1 & Contrôleur de gestion & 1 & Une heure & _- \\
\hline 6 & Ile-de-France & 3450 & 1 & Agent comptable & 2 & $\begin{array}{l}\text { Deux fois une } \\
\text { heure et demie }\end{array}$ & 6 mois \\
\hline 7 & Alpes & 550 & 1 & Contrôleur de gestion & 3 & $\begin{array}{l}\text { Une demi-heure } \\
\text { chacun }\end{array}$ & 4 mois \\
\hline 10 & Bourgogne & 520 & 2 & $\begin{array}{l}\text { Contrôleur de gestion } \\
\text { Directeur }\end{array}$ & 2 & $\begin{array}{l}\text { Une heure } \\
\text { Une demi-heure }\end{array}$ & 3 mois \\
\hline 11 & \begin{tabular}{|l} 
Provence-Alpes- \\
Côte d'Azur \\
\end{tabular} & 2550 & 2 & $\begin{array}{l}\text { Fondé de pouvoir } \\
\text { Contrôleur interne }\end{array}$ & 2 & $\begin{array}{l}\text { Deux fois une } \\
\text { heure et demie }\end{array}$ & 1 mois \\
\hline 12 & Pays de la Loire & 740 & 1 & Fondé de pouvoir & 1 & $\begin{array}{l}\text { Trois-quarts } \\
\text { d'heure }\end{array}$ & - \\
\hline 13 & Alsace & 600 & 2 & $\begin{array}{l}\text { Responsable Qualité } \\
\text { Agent comptable }\end{array}$ & 2 & $\begin{array}{l}\text { Une heure } \\
\text { Une heure et } \\
\text { demie }\end{array}$ & 2 mois \\
\hline 14 & Ile-de-France & 1460 & 1 & Contrôleur de gestion & 1 & $\begin{array}{l}\text { Deux fois une } \\
\text { heure }\end{array}$ & - \\
\hline
\end{tabular}

\section{Annexe 2}

Tableau 10. Tableau des valeurs propres (SPAD).

\begin{tabular}{|l|c|c|c|}
\hline Numéro de l'axe & Valeur propre & Pourcentage & Pourcentage cumulé \\
\hline 1 & 0.4426 & 19.67 & 19.67 \\
\hline 2 & 0.3384 & 15.04 & 34.71 \\
\hline 3 & 0.2909 & 12.93 & 47.64 \\
\hline 4 & 0.2387 & 10.61 & 58.25 \\
\hline 5 & 0.2344 & 10.42 & 68.67 \\
\hline 6 & 0.2131 & 9.47 & 78.14 \\
\hline 7 & 0.1799 & 7.99 & 86.13 \\
\hline 8 & 0.1692 & 7.52 & 93.65 \\
\hline 9 & 0.1428 & 6.35 & 100 \\
\hline
\end{tabular}


Tableau 11. Tableau des cosinus carrés et des contributions relatives.

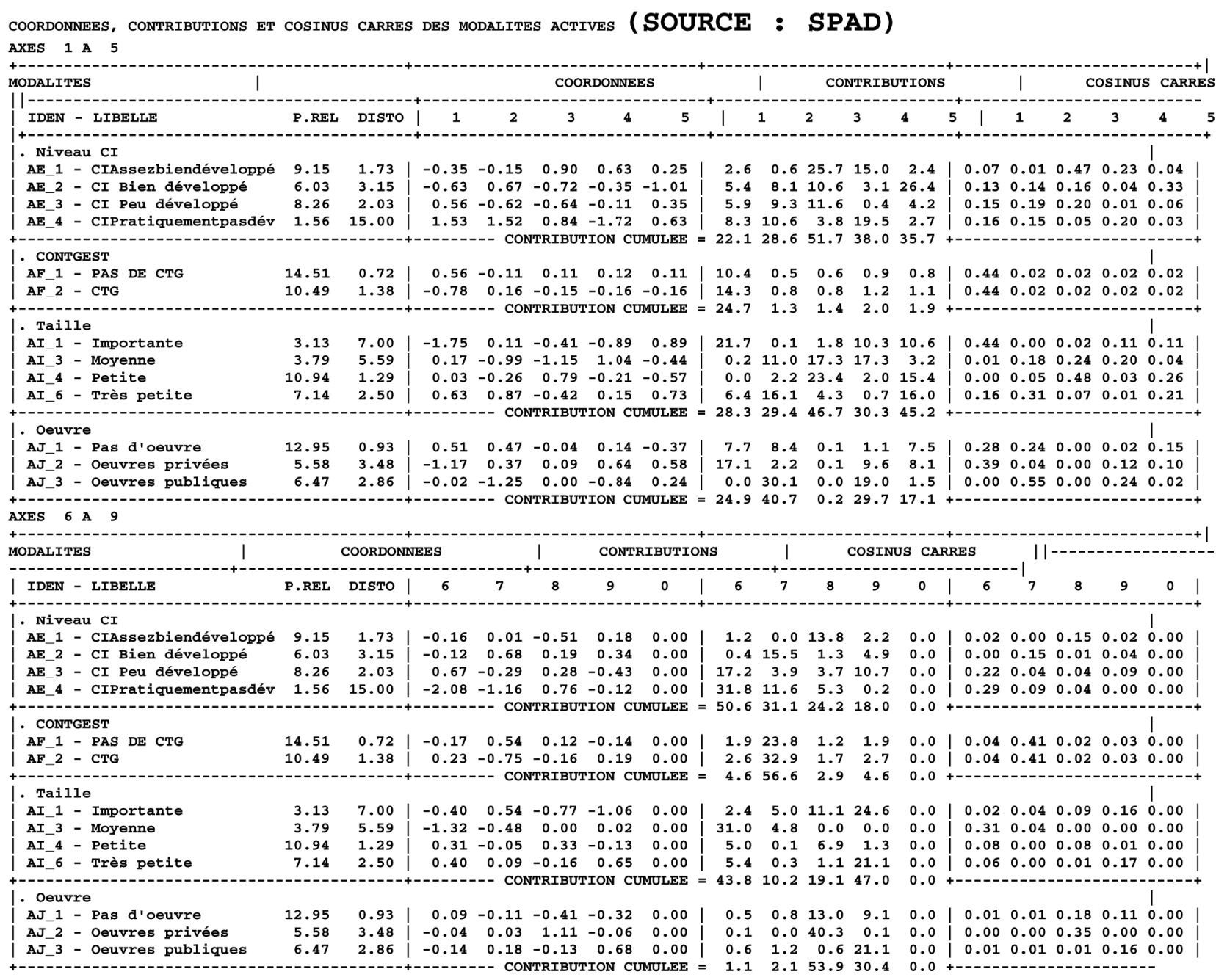

